\title{
FRACTALS AND THEIR APPLICATIONS: A REVIEW
}

\author{
Elizabeth K Joy, Dr. Vikas Garg \\ 20MSM2016@cuchd.in,Vikas.e9995@cumail.in
}

Department of Mathematics, University Institute of Sciences

Chandigarh University, Mohali-140413, India

\begin{abstract}
In this paper, I have discussed about fractals and their applications across various fields. The two key properties of fractals have been stated. A brief history about fractals is also mentioned. I have discussed about Mandelbrot fractal and have plotted it using python. The algorithm used is also given. A computer-generated fern is compared to a real fern to show how much fractals resemble the real-world objects. Various applications of fractal geometry have also been included. These include use of fractal concept in making devices that keeps chips cool. Other applications are in ecology, stock market and computer graphics.
\end{abstract}

\section{Keywords}

Fractals, Chaos, Recursive Formula, Mandelbrot Set, Barnsley's Fern, Computer Graphics, Stock Market, Ecology.

\section{Introduction}

A fractal is a never-ending pattern which is self-similar across different scales. These are made by repeating a basic cycle again and again in a continuous loop. "Fractals are images of dynamic systems-the pictures of chaos" [1]. Nature is full of fractals, trees around us, coastlines, rivers, seashells, are all examples of fractals. Fractals are known to have two key properties: first one is self-similarity, which means if we magnify the image many times, at every step we will eventually see the same pattern. Second is that fractals have a non-integer dimension. Fractals are generated using recursive sequences. It may not be as interesting at the starting but when iterated many times it is quite interesting. If we define terms of a sequence using one or more previous terms which are given, we call it a recursive/recurrence sequence. And the formula which relates the present term to the previous term is called the recursive formula. Let us consider an arithmetic sequence with a common difference of say 10 and the $8^{\text {th }}$ term is given to be 65 , and we need to find the $10^{\text {th }}$ term of the sequence, we use the formula $a n+1=a n+d$. We get, $a 9=a 8+10=75$ and $a 10=a 9+10=75+10=85$. Here $a n+1=a n+d$ is known as the recursive formula and the sequence generated is known as the recursive sequence. To generate a fractal, we need an initial point and then we follow a simple rule. It can be better understood 
with an example. Suppose we have a triangle of order 1 , to draw a triangle of order $\mathrm{n}$ we draw 3 triangles of order $n-1$ each having half the edge length of the triangle with order $n$. These 3 triangles of order $\mathrm{n}-1$ are placed in such a way that it forms the corners of the triangle with order $\mathrm{n}$. This is now repeated a million times to get a fractal. The fractal generated here is known as the Sierpinski Triangle, it has a dimension $=1.585$.

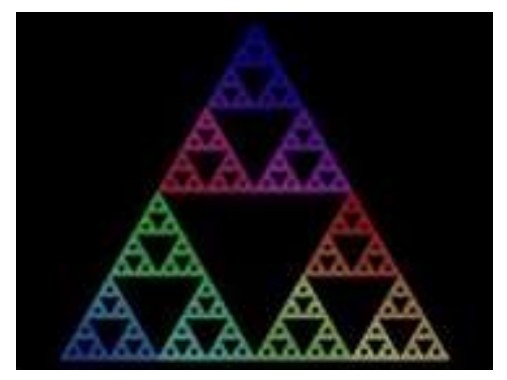

Another beautiful fractal I studied about is the fractal generated by the factorial function [2]. Consider the factorial function, $\mathrm{n}$ ! (Read as $\mathrm{n}$ factorial). Where $n !=n(n-1)(n-2) \ldots .(3)(2)(1)$ : this is also an example of a recursive function. This is iterated a million times using a computer to get a set of points. These points are then plotted to generate beautiful looking fractals.

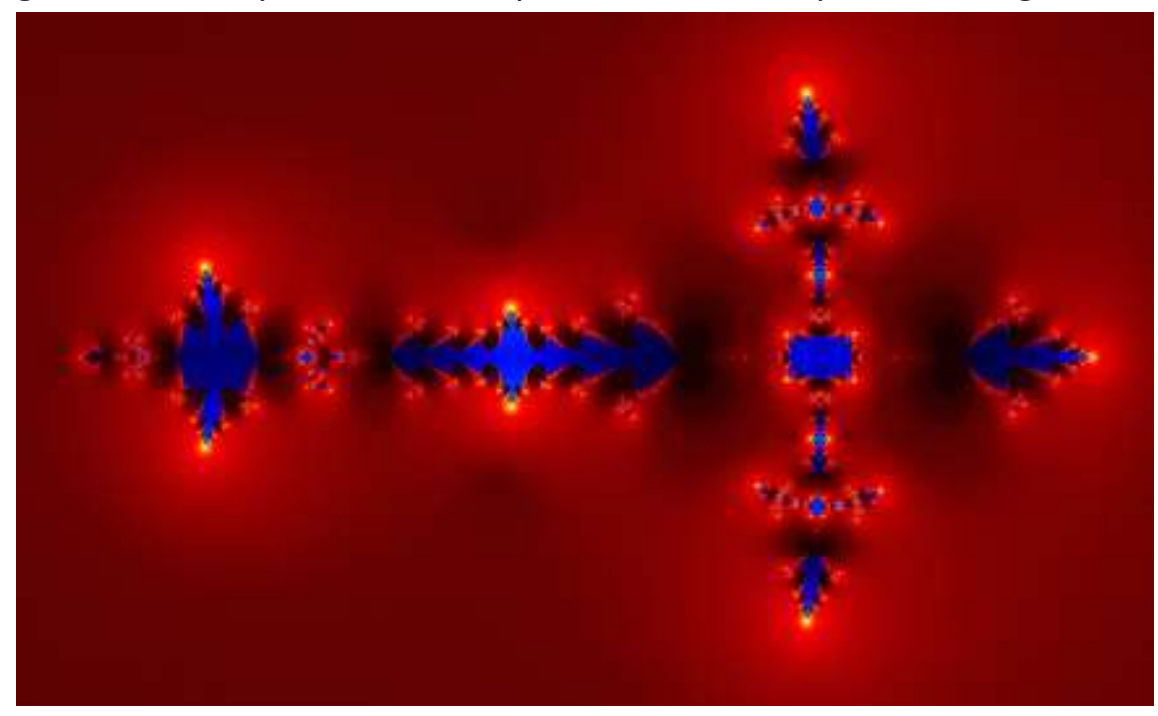

\section{History}

In the $20^{\text {th }}$ century, scientists faced a new revolution called the chaos [5]. Chao has its origin from an old Greek word 'haino' which means open widely. Currently this word is used as a synonym for mess and lack of order. Nature rarely exhibits geometric order. The objects and forms found in nature are irregular and chaotic. These are the forms that are known as fractals.

In the 1940s British scientist Louis Richardson had observed that there can be great variation between different measurements of a coastline, he said that it depends on how long a yardstick is and how much patience a person has. If the coastline of Britain is measured with a one-mile 
yardstick then so many yardsticks would be used which gives so many miles, if a one-foot yardstick is used, it turns out to be longer. And every time a shorter yardstick is used; a longer number is observed because one can always find finer indentations.

Mandelbrot saw that the finer and finer the indentation gets, it was precisely what was needed to model coastlines. According to Mandelbrot a coastline in geometric terms is a fractal and though he knew he couldn't measure its length but its roughness. In normal geometry we think of one dimension as a straight line, two dimensions as something that has a surface area, and three dimensions as something that has volume. But what if some figures have a dimension between say 1 and 2; these are the fractals and the rougher they are the higher their fractal dimension. A French mathematician Gaston Julie in the era of the first world war looked at what happens when we take a simple equation and iterate it through a feedback loop that means we take a number put it in the formula we get a number then again, this number is put in the formula and another number is obtained and, in this way, if we keep iterating it repeatedly, we get a series of numbers called a Julia set.

But iterating it a thousand times by hand was not possible so it had to wait till the first computers were invented. At IBM, Mandelbrot used a computer to run the equation millions of times. He then plotted these points on a graph to obtain hundreds of images. In 1980, he then created an equation of his own, one that combined all the Julia sets into one image. When he iterated his equation, he got his own set of numbers which he then graphed on a computer to obtain an image which was kind of a road map of all the Julia set and it became the emblem of fractal geometry.

Consider the recursive formula $z n=z n-12+c$, where $c$ is any complex number. All those values of $c$ for which the orbit of the critical point $z=0$ under the iteration of the above-mentioned formula remains bounded makes the Mandelbrot set.

Let $c=1$, the sequence generated will be $0,1,2,5,26, \ldots$ which tends to infinity so 1 does not belong to the Mandelbrot set. Now let $\mathrm{c}=-1$, the sequence generated is $0,-1,0,-1,0, \ldots$ which is bounded, so -1 belongs to the set. So, in general we can say that $\mathrm{c}$ is the set of all complex numbers such that $f c(z)=z 2+c$ does not diverge when iterated from $z=0$, that means the sequence $f c(0), f c(f c(0)) \ldots . .$. remains bounded in absolute value.

\section{I have used python language to generate a fractal and plot it.}

\section{Algorithm:}




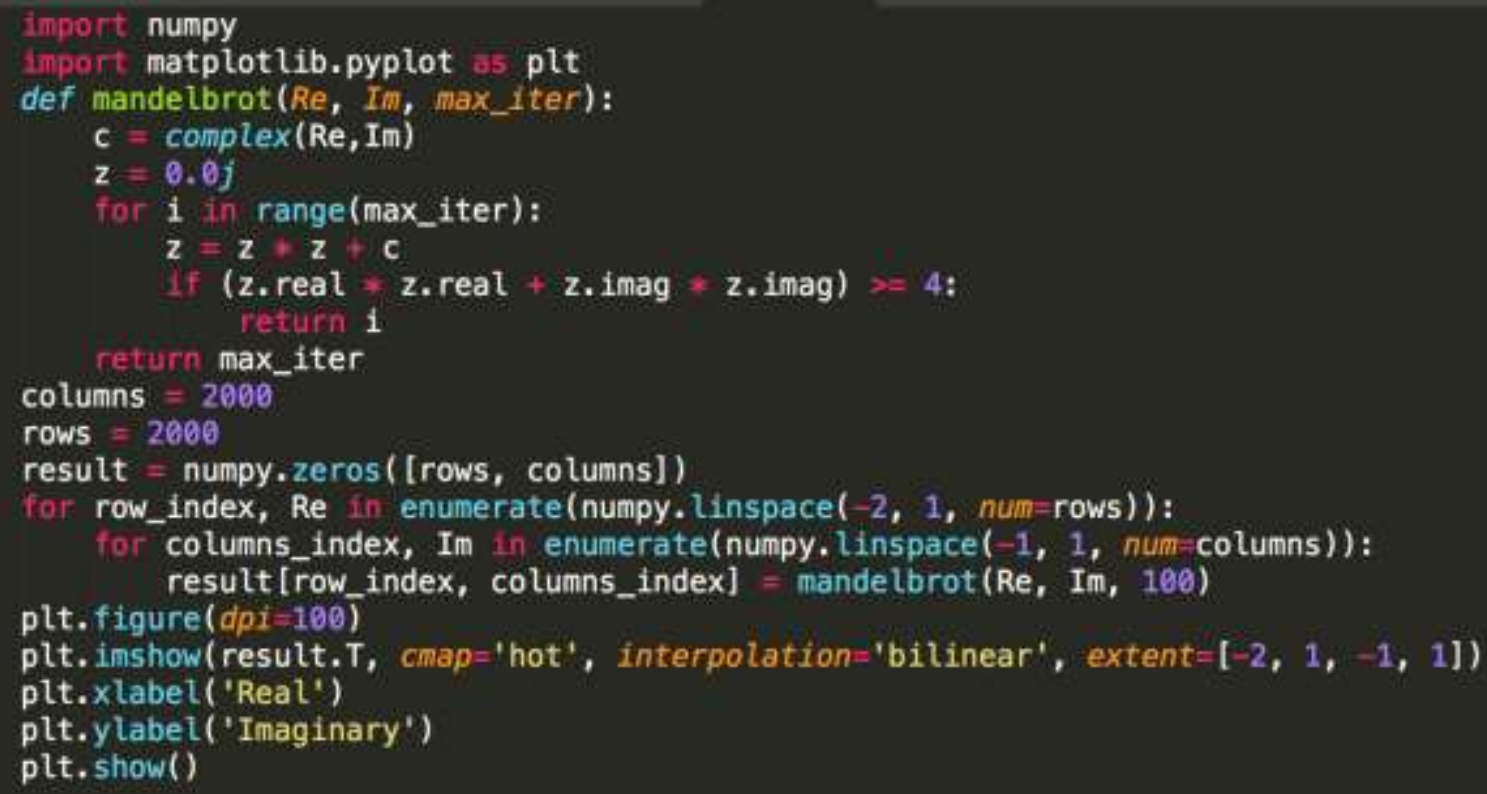

\section{Output:}

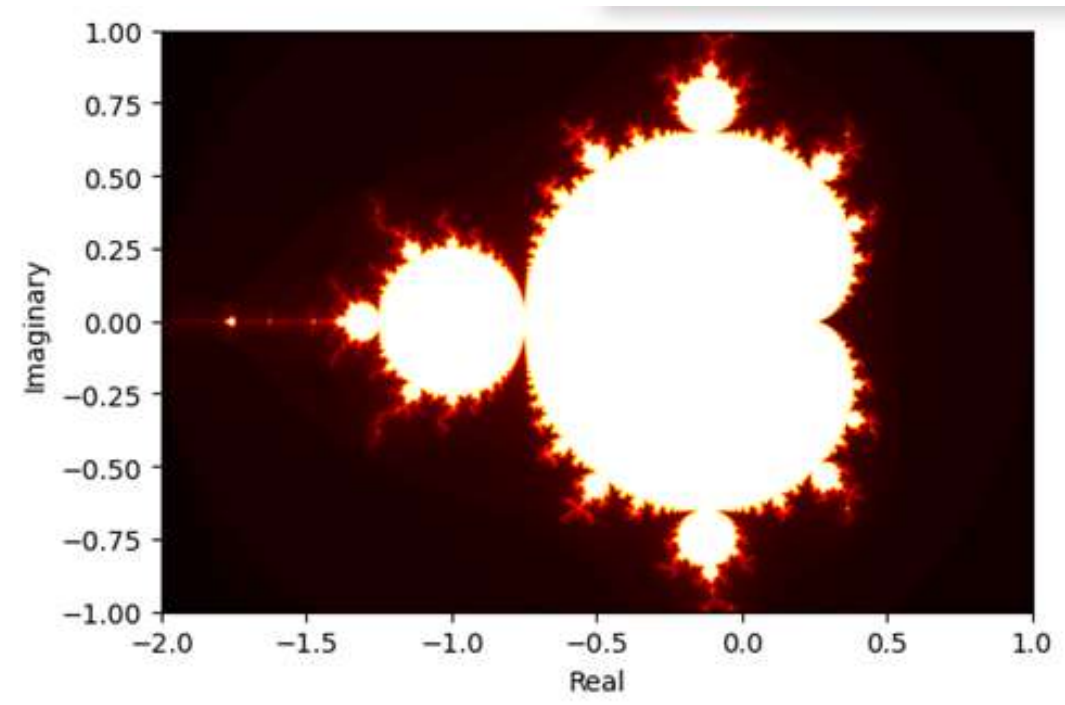

\section{Comparing a computer-generated fractal to a real fern}

It is really amazing to see how much a computer-generated fractal resembles the real-life objects. Barnsley's Fern is a fractal first generated by British mathematician Michael Barnsley in his book 'Fractals everywhere'. The fern is one of the fundamental instances of self-similar sets, it is a numerically produced design that can be reproducible at any amplification. The fern code created by Barnsley is an illustration of an iterated function system (IFS) to make a fractal. He has utilized fractals to show an assorted scope of marvels in science and innovation. IFSs provide models for certain plants, leaves, and ferns, by excellence of the self-comparability which 
regularly happens in fanning structures in nature. Yet, nature likewise shows irregularity and variety starting with one level then onto the next; no two plants are actually similar, and the branching fronds become leaves at a more limited size.

It is hypothesised that if the fractal model is found to have a good match with the geometry of the plant, then it can be used to retrieve information stored in the genes of that plant.

\section{Generating Barnsley Fern with an online generator [3]:}

Barnsley used the following transformation

$$
\mathrm{f}(\mathrm{x}, \mathrm{y})=\left[\begin{array}{ll}
a & b \\
c & d
\end{array}\right]\left[\begin{array}{l}
x \\
y
\end{array}\right]+\left[\begin{array}{l}
e \\
f
\end{array}\right]
$$

Values are given in the table bellow

\begin{tabular}{|c|c|c|c|c|c|c|}
\hline a & b & C & d & & $f$ & $p$ \\
\hline 0 & 0 & 0 & 0.16 & 0 & 0 & 0.01 \\
\hline 0.85 & 0.04 & -0.04 & 0.85 & 0 & 1.6 & 0.85 \\
\hline 0.2 & -0.26 & 0.23 & 0.22 & 0 & 1.6 & 0.07 \\
\hline-0.15 & 0.28 & 0.26 & 0.24 & 0 & 0.44 & 0.07 \\
\hline
\end{tabular}

Following figure is of the fractal generated when iterated for 1000000 times. 


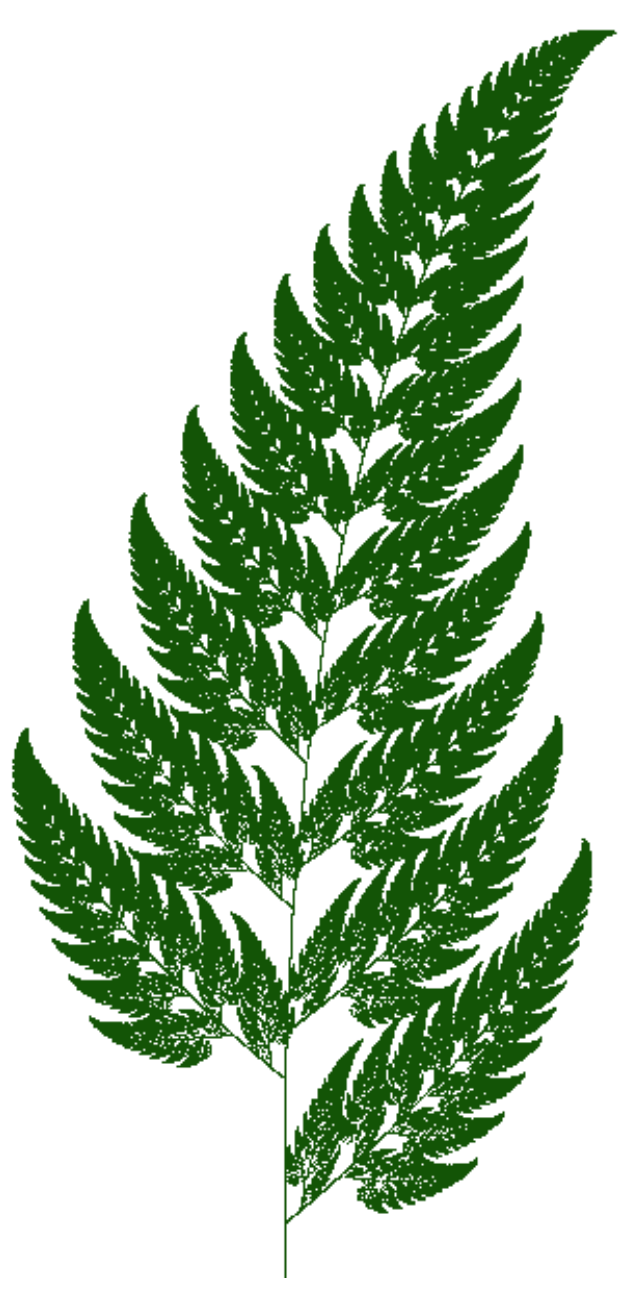

If we magnify this image repeatedly, we get the same pattern again and again. The estimated dimension of the Barnsley Fern is 1.45 .

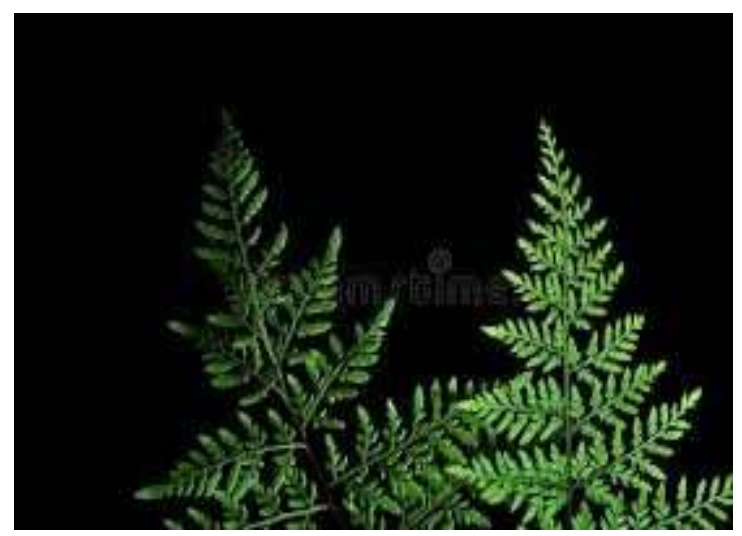

This is an actual picture of a fern plant. If we compare it with the fractal generated above; both are almost identical. I have tried to portray it using the following image. I have replaced one of the leaves with the computer-generated leaf. If we replace each of the leaf of the fern with a fractal image, it will look like the fern itself. 


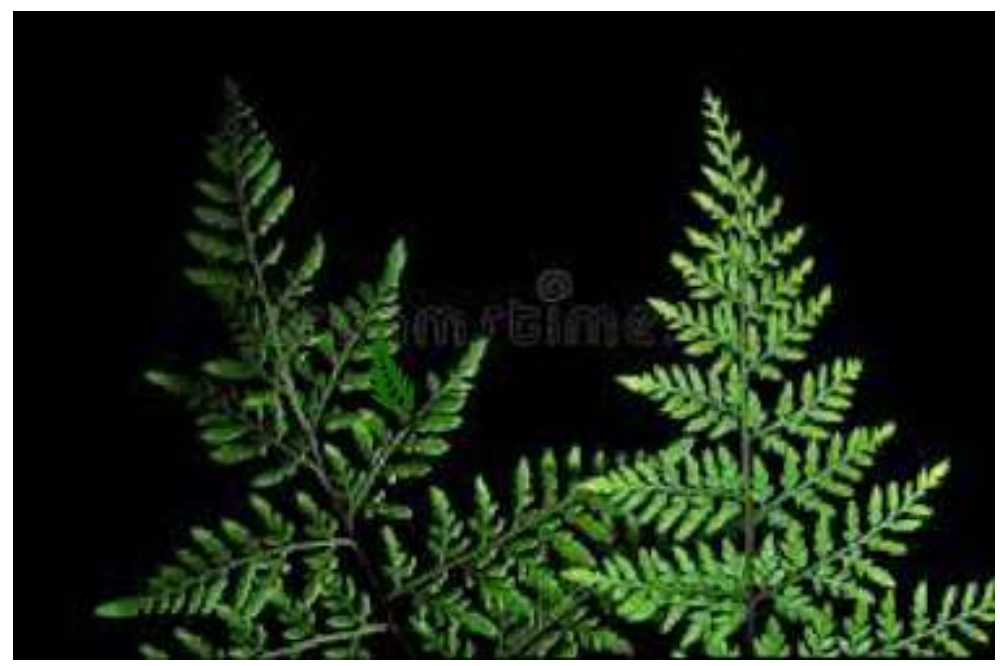

\section{Applications of Fractals}

\section{Fractal Device}

Engineers are utilizing the thoughts of fractal calculation in an assortment of uses. Regularly we are confronted with an errand that is like something that nature has effectively discovered an answer for. The derivation of inspiration from nature to build human designs is called biomimicry. Small and faster computers tend to produce more heat, which should be disseminated or, in all likelihood the PCs will overheat and break. Students at Oregon State University have created fractal design that can be scratched into a silicon chip to permit a cooling liquid, (for example, fluid nitrogen) to consistently stream across the outside of the chip and keep it cool. The fractal design which they got from our veins gives a basic low-constrain organization to achieve this undertaking without any problem [8].
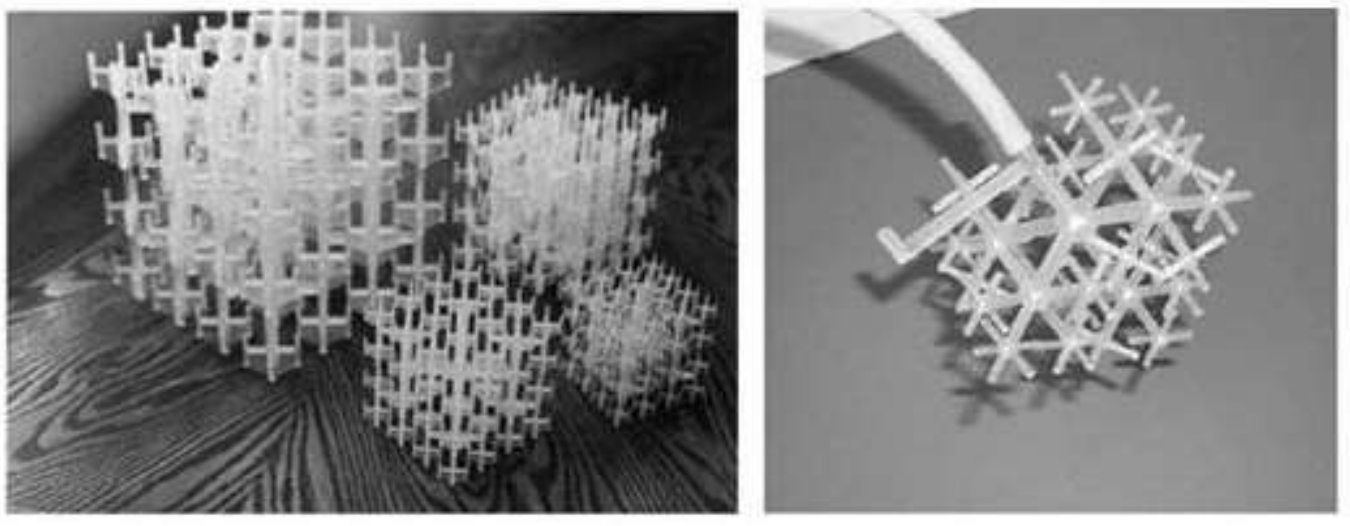

[8]

Fractals can be found in economically accessible receiving wires, delivered for applications, for example, cellphones and WIFI. The self-similar structure of fractal antennas enables them to get and transmit over a range of frequencies, allowing powerful antennas to be made more conservative.

\section{In Ecology}


One of the more direct uses of the ideas of fractal is to the problem of measuring available habitat [7]. They applied fractal methods to the question of why in a given habitat there tend to be so many more individuals of small animals than of larger ones. Utilizing photos of different kinds of vegetation, they compute a value for the fractal measurement of the habitat flora by boundary-grid strategy. They found that organisms that are an order of magnitude smaller in length would have 3.16 to 10 times more available living space. They noticed that fractal scaling of the vegetational substrate, which gives little arthropods much more living space than is accessible to bigger ones on a similar substrate, is subjectively predictable with expectations of individual plenitude dependent on allometric contentions. Fractals may give a strategy for acquiring target answers to such troublesome issues in order hypothesis as how to decide limits between progressive levels and how to decide the scaling rules for extrapolating inside each level.

\section{In stock market prediction.}

Mandelbrot, in his book [6] states that stock market indices follow a fractal pattern. There are many methods for predicting the shifts when the market is stable but when it is unstable there was no methods available. Using the fractal dimension and Hurst exponent we can predict the shift in stock market indices. As mentioned earlier the stock market is a fractal. If we identify the fractal pattern and calculate the fractal dimension, we can find the Hurst exponent using the formula $H=2-D$. If $\mathrm{H}$ comes out to be less than 0.5 , the time series display Anti persistence, that is if the current stock price is high, in the next period it will go down, and vice-versa. If $\mathrm{H}>0.5$ then the series is persistent that means it will remain high if it was high in the previous period and will remain low if it was low in the previous period. The prediction cannot be made if $\mathrm{H}=0.5$.

\section{In computer graphics}

Computer graphics means to draw pictures on computer screens by computation, creation and manipulation of data. Many of the mathematical techniques have found a firm place in computer graphics for creating stunning images as well as natural looking images. In most general sense fractals can be used in structures having symmetric scale. For example, mipmapping, quad trees, oct trees and things like binary search partition trees are all scale symmetric, they allow details to be stored at different scales in the same way. Some movies like big hero, Dr. Strange and suicide squad render variations of the 3D Mandelbulb and Mandlebox fractals [5].

\section{Conclusion}

Fractals are beautiful self-similar structures found everywhere in nature. Most of the forms in nature like the trees, clouds, coastlines, ferns etc seem to have a fractal pattern. In this paper we have seen how much a computer-generated fractal can resemble a real-world object. So, studying the fractal nature of objects and determining its dimension prove to be helpful in analysing the forms in nature. Talking about the uses of fractals, we see that they define images that cannot be defined by Euclidean Geometry. It is used in arts; fractal art is considered to be true art. Fractals are a very important part in biological studies. Another most important use is with regards to image compressing. The Mandelbrot set is also having its uses in public key cryptography [4]. RSA 
utilizes secluded dramatic idea from number theory to create the public key and private key sets. This set is generated using the Mandelbrot sets.

\section{References}

[1] Ada Knowe,2018, Introduction to fractals, Bit Nibble Byte.

[2] Thomas Oléron Evans,2015, Fractal Factorials, Mathistopheles.

[3] A Barnsley Fern Generator, https://www.chradams.co.uk/fern/maker.html

[4] S. Kumar, "Public Key Cryptography System Using Mandelbrot Sets",MILCOM 2006-2006 IEEE Military Communications conference,2006, pp. 1-5,doi:10.1109/MILCOM.2006.302396.

[5] L. M .Kocic,"Fractals And Their Applications In Computer Graphics",FILOMAT 9:2(1995),207231.

[6] Mandelbrot B.And Richard L. Hudson. "The misbehaviour of Markets: A fractal View of Risks, Ruin,And Reward".(2004).

[7] George Sugihara,Robert M. May, "Applications of Fractals in ecology", Trends in Ecology \& Evolution, Volume 5,Issue 3,1990,pages 76-86.

[8] Huang, Zhiwei et al."Review of Fractal Heat Exchangers".(2016). 